\title{
BARBARA WEINSTOCK LECTURES ON THE MORALS OF TRADE
}

This series will contain essays by representative scholars and men of affairs dealing with the various phases of the moral law in its bearing on business life under the new economic order, first delivered at the University of California on the Weinstock foundation. 
